www.nature.com/jhg

\title{
Mutation spectrum of MMACHC in Chinese patients with combined methylmalonic aciduria and homocystinuria
}

\author{
Mei-Ying Liu ${ }^{1}$, Yan-Ling Yang ${ }^{2}$, Ying-Chen Chang ${ }^{3}$, Szu-Hui Chiang ${ }^{4}$, Shuan-Pei Lin ${ }^{5}$, Lian-Shu Han ${ }^{6}, \mathrm{Yu} \mathrm{Qi}^{7}$, \\ Kwang-Jen $\mathrm{Hsiao}^{4,8}$ and Tze-Tze $\mathrm{Liu}^{3,8}$
}

The $c b / C$ type of combined methylmalonic aciduria (MMA) and homocystinuria (HC) is the most common inborn error of vitamin $\mathrm{B}_{12}$ metabolism and is caused by mutations in the $M M A C H C$ gene. To elucidate the spectrum of mutations that causes combined MMA and $\mathrm{HC}$ in Chinese patients, the MMACHC gene was sequenced in 79 unrelated Chinese patients. Sequence analysis identified $98.1 \%$ of disease alleles and found that all patients had at least one MMACHC mutation. A total of 24 mutations were identified. Out of the 24 mutations identified, 9 were novel ones, including missense mutations (c.365A $>T$ and c.452A $>G$ ), nonsense mutations (c.315C $>G$ and c.615C $>A$ ), deletions (c.99delA and c.277-3_c.303del30), duplications (c.248dupT and c.626dupT) and an insertion (c.445_446insA). The c.609G $>A$, c.658_660delAAG, c.482G $>A$, c. 394C $>T$ and c.80A $>G$ mutations were the most common mutations and accounted for $80 \%$ of disease alleles. Haplotype analysis suggests that the spread of the c.80A $>$ G, c.609G $>A$ and c.658_660delAAG mutations in Chinese patients were caused by a founder effect. The results indicate that defects occurring in the MMACHC gene are the major cause of this disease in Chinese patients with combined MMA and $\mathrm{HC}$, and direct mutation analysis can therefore be used as a rapid confirmatory diagnosis among these Chinese patients.

Journal of Human Genetics (2010) 55, 621-626; doi:10.1038/jhg.2010.81; published online 15 July 2010

Keywords: $c b / C$; Chinese; founder; methylmalonic aciduria and homocystinuria; MMACHC

\section{INTRODUCTION}

Methylmalonic aciduria (MMA) is a heterogeneous autosomal recessive metabolic disorder of methylmalonate and cobalamin (vitamin $\mathrm{B}_{12}$ ), which encompasses at least seven different complement groups and is caused by a defect of methylmalonyl CoA mutase (MCM, EC 5. 4.99.2) apoenzyme or by one of a number of other defects in the biosynthesis of adenosylcobalamin (AdoCbl). ${ }^{1}$ In mammals, cobalamin is ingested in the diet and is converted into two active coenzymes, AdoCbl in the mitochondria and methylcobalamin ( $\mathrm{MeCbl})$ in the cytosol, which are required for the metabolism of methylmalonic acid and homocysteine, respectively. AdoCbl and $\mathrm{MeCbl}$ are the coenzymes for MCM and methionine synthase (MS, EC 2.1.1.13), respectively. Deficiencies in the biosynthesis of AdoCbl, namely cblA (MIM 251100), cblB (MIM 251110) and cblD-variant 2 (MIM 277410), have been shown to impair mitochondrial MCM activity and these patients present with only MMA. ${ }^{1} c b l \mathrm{E}$ (MIM 236270), cblG (MIM 250940) and cblD-variant 1 (MIM 277410), with defects in the biosynthesis of $\mathrm{MeCbl}$ in the cytosol, have defective remethylation of homocysteine to form methionine and are characterized by homocystinuria (HC) only. ${ }^{1}$ Three different genetic defects in early cobalamin metabolism, namely cblF (MIM 277380), cblC (MIM 277400) and $c b l$ D (MIM 277410), have been found to cause combined MMA and HC. ${ }^{1}$

Type $c b l C$ MMA is one of the most common inherited disorders of vitamin $B_{12}$ metabolism, and two distinct phenotypes have been defined in terms of age of onset. ${ }^{2}$ Early-onset patients present in the first year of life with nonspecific systemic, neurological and hematological abnormalities and the survivors may have severe neurological impairment. $^{2}$ Late-onset patients have symptoms that usually involve acute neurological deterioration and this occurs after the age of 4 years. These late-onset patients have a better outcome after treatment. $^{2-6}$

The MMACHC gene is responsible for $c b l \mathrm{C}$ MMA and was identified by Lerner-Ellis et al. ${ }^{7}$ The MMACHC gene is located on chromosome 1 and comprises four coding exons. The gene encodes a protein of 282 amino acids. Recently, Kim et al. ${ }^{8}$ suggested that the

\footnotetext{
${ }^{1}$ Institute of Genetics, National Yang-Ming University, Taipei, Taiwan; ${ }^{2}$ Department of Pediatrics, Peking University, First Hospital, Beiijing, China; ${ }^{3}$ Genome Research Center, National Yang-Ming University, Taipei, Taiwan; ${ }^{4}$ Department of Medical Research and Education, Taipei Veterans General Hospital, Taipei, Taiwan; ${ }^{5}$ Departments of Pediatrics and Medical Research, Mackay Memorial Hospital and Mackay Medicine, Nursing and Management College, Taipei, Taiwan; ${ }^{6}$ Department of Pediatric Endocrinology and Genetic Metabolism, Shanghai Institute for Pediatric Research, Xinhua Hospital, Shanghai Jiaotong University School of Medicine, Shanghai, China; ${ }^{7}$ Central Laboratory, Peking University, First Hospital, Beijing, China and ${ }^{8}$ Department of Education and Research, Taipei City Hospital, Taipei, Taiwan

Correspondence: Dr T-T Liu, Genome Research Center, National Yang-Ming University, No.155, Sec. 2, LiNong Street, Taipei, Taiwan 112 , ROC.

E-mail: ttliu@ym.edu.tw
}

Received 10 February 2010; revised 21 May 2010; accepted 4 June 2010; published online 15 July 2010 
human MMACHC catalyzes the reductive decyanation of cyanocobalamin and may serve as a trafficking chaperone for intracellular cobalamin.

To date, 57 different mutations in the MMACHC gene have been reported. ${ }^{7,9-13}$ Mutation analysis of $c b l \mathrm{C}$ patients has shown that the c.271dupA mutation accounts for $40-55 \%$ of all disease alleles in European populations. ${ }^{7,11}$ Several ethnic-specific mutations have also been observed, including the c.331C $>$ T mutation in Cajun, FrenchCanadian, Italian and Portuguese and the c.394C $>\mathrm{T}$ mutation in Asiatic-Indian, Pakistani, Middle Eastern, Italian and Portuguese. ${ }^{7,11,14}$ In Hong Kong, one $c b l \mathrm{C}$ patient has been also identified as having a compound heterozygous genotype composed of the c.394C $>\mathrm{T}$ and c.536_537insAT mutations. ${ }^{10}$ In addition, among East Asians, the c.609G $>$ A mutation has also been observed. ${ }^{7}$

MMA is the most common symptomatic organic acidurias in China, ${ }^{15}$ but the precise incidence of Chinese population remains unclear. In 2006, Yang et al. ${ }^{16}$ reported that the incidence of patients with combined MMA and HC among MMA patients was higher in China than in other countries, suggesting that combined MMA and $\mathrm{HC}$ might be more common in Chinese populations. However, in Taiwan, 14 MMA patients were detected in the expanded newborn screening program from 1321123 newborns, including 13 mut-type MMA and $1 \mathrm{cblC}$-type MMA, ${ }^{17}$ which indicates that the mut-type MMA is more common than the cblC-type MMA in Southern Chinese. To study the molecular defects responsible for combined MMA and HC among Chinese patients, we performed a molecular genetic analysis and examined the mutation spectrum of the MMACHC gene across 79 Chinese patients with combined MMA and $\mathrm{HC}$.

\section{SUBJECTS AND METHODS}

\section{Subjects}

In this study, 79 unrelated patients (47 males and 32 females) suspected as having combined MMA and HC were clinically identified. The patients were from Beijing $(n=75),{ }^{16,18-19}$ Taiwan $(n=2)$ and Shanghai $(n=2)$. Most patients (75 of 79) were Northern Chinese. All patients were born from non-consanguineous families. Patients were diagnosed as having elevated urinary methylmalonate using gas chromatography mass spectrometry. Elevated total homocysteine (tHcy) levels in blood or urine specimens were also detected in 78 out of the 79 patients. The tHCy level in the blood or urine of the remaining one patient was not determined before the patient's death. However, elevated methylmalonate, methylcitrate and tHcy in the amniotic fluid of an affected sibling of this family were detected. ${ }^{19}$ Patients and parents were informed about the study and consented to participate. Patients with clinical presentations that appeared in the first year of life were classified as early-onset patients, and patients with symptoms that presented after the age of 4 years were classified as late-onset patients. ${ }^{2}$ To determine the allelic frequency of sequence variations in the general Chinese population, control samples were collected from 70 anonymous Chinese controls. These controls are apparently normal and had no medical history associated with disorders of methylmalonate metabolism.

\section{Mutation analysis}

Genomic DNA was isolated from peripheral leukocytes, cultured fibroblasts or lymphoblasts using standard methods. The method for genomic DNA isolated from a dried blood spot collected on filter paper has been described elsewhere. $^{20-21}$ The whole coding region sequence and exon-intron boundary sequence of the MMACHC gene were PCR amplified using primer sets for exons 1, 2, 3 and 4 a as described by Lerner-Ellis et al. ${ }^{7}$ For patients with only one mutation identified in the coding exons and flanking sequence, sequence analysis was extended into the $3^{\prime}$-untranslated region (exons $4 \mathrm{~b}, 5 \mathrm{a}$ and $5 \mathrm{~b}$ in Lerner-Ellis et al. ${ }^{7}$ ). The four coding exons and exon-intron boundary sequence of the MMACHC gene were also sequenced in 70 anonymous Chinese controls.
The PCR products were sequenced directly by thermocycle sequencing using the BigDye terminator v3.1 sequencing kit (Applied Biosystems, Foster City, CA, USA) and analyzed using an ABI 3730XL DNA Analyzer (Applied Biosystems). The sequences obtained were analyzed by the Polyphred/Phrap/ Consed system (http://www.phrap.org/). ${ }^{22-23}$ The mutation nomenclature follows the recommendation from den Dunnen and Antonarakis ${ }^{24}$ using GenBank accession no. NM_015506.2 as the reference sequence. The numbering of the cDNA nucleotide +1 is the A of the ATG translational initiation codon. For mutations that were not reported in the MMACHC gene, the alterations were confirmed by sequencing the second PCR products. Disease-causing mutations were confirmed by studying the prevalence of these mutations among the general Chinese controls, linkage analysis in the families, concurrent appearance of the mutations and/or the conservation of amino acid affected.

\section{Haplotype analysis}

One nearby microsatellite marker and four intragenic single-nucleotide polymorphisms (SNPs) were studied to determine the haplotype of the recurrent mutations. A short tandem repeat marker D1S2677, which is located $173 \mathrm{~kb}$ upstream to the MMACHC gene, was genotyped using the ABI 3730 DNA Analyzer. The allelic frequencies were estimated from 257 control subjects.

The SNPs used were (from $5^{\prime}$ to $3^{\prime}$ ): $\operatorname{rs} 3748643$ (c.1-302T $>$ G, $5^{\prime}$-untranslated region), rs3748644 (c.1-234A > G, 5'-untranslated region), c.277-49A > G and rs2275276 (c.321G >A, p.V107V, exon 3). The GENECOUNTING software (version2.1) 25-26 $^{25}$ was used to estimate the frequencies of the haplotypes of the SNPs from 70 normal controls. A $\chi^{2}$-test with or without Yates' correction was used to assess the difference between the distribution of the normal and mutant alleles with a $P$-value $<0.01$ considered to be significant.

\section{RESULTS AND DISCUSSION}

In this study, 79 unrelated patients were studied by mutation analysis. Sequencing analysis identified MMACHC mutations in 98.1\% (155 of 158) of all disease alleles; all together, only three patients had one of their disease mutations unidentified (Supplementary Table S1). The results thus indicate that a defect in the MMACHC gene is the major cause of disease among Chinese patients with combined MMA and HC.

The mutations identified in this study, their predicted effects on proteins and the allelic frequency of each mutation, are shown in Table 1 and Figure 1. A total of 24 mutations were identified in the coding exons and exon-intron boundaries; these mutations include 5 missense, 7 nonsense, 5 deletions, 4 duplications, an insertion, 1 potential splice-site mutation and 1 point mutation that may affect the translational initiation codon (Table 1). Among these 24 mutations, 9 were novel, whereas the remaining 15 mutations have been reported previously. ${ }^{7,9,12-13}$ The nine novel mutations (Table 1) include two missense mutations: c.365A $>$ T (p.H122L) and c.452A $>$ G (p.H151R), two nonsense mutations: c.315C $>\mathrm{G}$ (p.Y105X) and c.615C > A (p.Y205X), two duplications: c.248dupT (p.A84GfsX8) and c.626dupT (p.T210DfsX35), one insertion: c.445_446insA (p.C149X) and two deletions: c.99delA (p.E33DfsX43) and c.277-3_ c.303del30 (p.S93_Q143del, (r.spl?)). None of these mutations were found in 140 normal control alleles.

Of the two novel missense mutations, the c.365A > T (p.H122L) mutation was predicted to replace a histidine (polar and charged) with a leucine (aliphatic and hydrophobic), whereas the c.452A $>\mathrm{G}$ (p.H151R) mutation substituted an arginine (polar and charged) for a histidine. Both histidine residues are located in the putative $\mathrm{B}_{12}$-binding domain (Figure 1) of the enzyme, ${ }^{7}$ a region that is highly conserved in all orthologs across eight different species (such as Homo sapiens, Pan troglodytes, Mus musculus, Bos taurus, Gallus gallus, Danio rerio, Xenopus tropicalis and Caenorhabditis elegans). One mutation, namely c.365A $>\mathrm{G}$, resulting in an alteration to the same amino acid (p.H122R), was previously described in two patients. ${ }^{6}$ These results 
Table 1 MMACHC mutations identified in 79 Chinese patients with combined MMA and HC

\begin{tabular}{|c|c|c|c|c|}
\hline Mutation type & Mutation $^{\mathrm{a}}$ & Predicted effect on protein & Allele no. & Reference \\
\hline \multirow[t]{5}{*}{ Missense } & c. $347 \mathrm{~T}>\mathrm{C}$ & p.L116P & 1 & Lerner-Ellis et al. ${ }^{7}$ \\
\hline & c. $365 A>T$ & p.H122 L & 2 & This study \\
\hline & $c .452 A>G$ & p.H151R & 1 & This study \\
\hline & c. $482 \mathrm{G}>\mathrm{A}$ & p.R161Q & 11 & Lerner-Ellis et al. ${ }^{7}$ \\
\hline & c. $616 \mathrm{C}>\mathrm{T}$ & p.R206W & 1 & Lerner-Ellis et al. ${ }^{7}$ \\
\hline \multirow[t]{7}{*}{ Nonsense } & c. $217 \mathrm{C}>\mathrm{T}$ & p.R73X & 1 & Lerner-Ellis et al. ${ }^{7}$ \\
\hline & c. $315 \mathrm{C}>\mathrm{G}$ & p.Y105X & 2 & This study \\
\hline & c. $394 \mathrm{C}>\mathrm{T}$ & p.R132X & 9 & Lerner-Ellis et al. ${ }^{7}$ \\
\hline & c. $457 \mathrm{C}>\mathrm{T}$ & p.R153X & 1 & Lerner-Ellis et al. ${ }^{7}$ \\
\hline & c. $481 C>T$ & p.R161X & 1 & Lerner-Ellis et al. ${ }^{7}$ \\
\hline & c. $609 \mathrm{G}>\mathrm{A}$ & p.W203X & 76 & Lerner-Ellis et al. ${ }^{7}$ \\
\hline & c. $615 \mathrm{C}>\mathrm{A}$ & p.Y205X & 1 & This study \\
\hline \multirow[t]{5}{*}{ Deletion } & c.99delA & p.E33DfsX43 & 1 & This study \\
\hline & c.146_154del9 & p.A49_V52delinsV & 1 & Tang et al. ${ }^{13}$ \\
\hline & c.277-3_303del30 & p.S93_Q143del (r.sp/?) & 1 & This study \\
\hline & c.398_399delAA & p.Q133RfsX5 & 1 & Lerner-Ellis et al. ${ }^{7}$ \\
\hline & c.658_660delAAG & p.K220del & 22 & Lerner-Ellis et al. ${ }^{7}$ \\
\hline \multirow[t]{4}{*}{ Duplication } & c.248dupT & p.A84GfsX8 & 1 & This study \\
\hline & c.271dupA & p.R91KfsX14 & 1 & Lerner-Ellis et al. ${ }^{7}$ \\
\hline & c.567dupT & p.I190YfsX13 & 6 & Lerner-Ellis et al. ${ }^{7}$ \\
\hline & c.626dupT & p.T210DfsX35 & 1 & This study \\
\hline Insertion & c.445_446insA & p.C149X & 1 & This study \\
\hline Splicing & c. $80 A>G$ & p.Q27R $[r .(s p / ?)]$ & 8 & Lerner-Ellis et al. ${ }^{7}$ \\
\hline Initiation codon change & c. $1 A>G$ & p.M1? & 4 & Heil et al. ${ }^{9}$ \\
\hline
\end{tabular}

Abbreviations: $\mathrm{HC}$, homocystinuria; MMA, methylmalonic aciduria.

a Mutations newly described in this study are shown in bold type.

a

Chromosome $1 \mathrm{p} 34.1$

Telomere

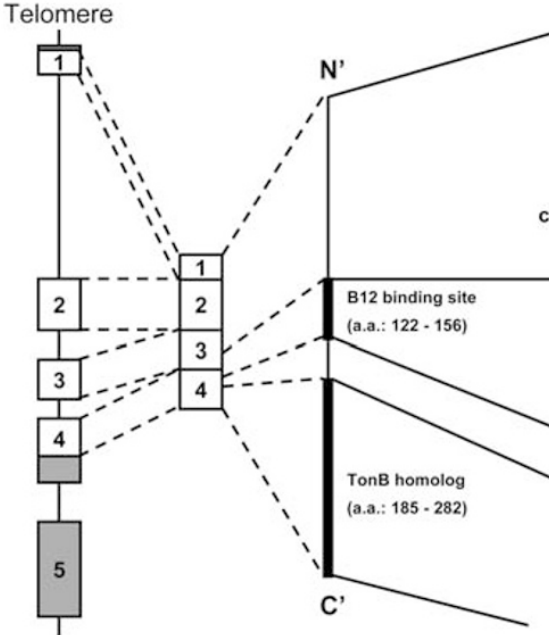

Centromere b c

Mutation

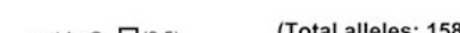

c. $80 A>G \square(2.5)$

c.80A>G $\square(5.1)$

c. 146 . 154 delA $(0.6)$

_154del9 (0.6)

c.217C>T (0.6)

c.271dupA $(0.6)$

.277-3_c.303del30 $(0.6)$

c. $315 \mathrm{C}>\mathrm{G}(1.3)$

C. $347 \mathrm{~T}>\mathrm{C}$

c.365A >T (1.3)

c.398 399delAA $(0.6)$

c.445_446insA $(0.6)$

c. $452 A>G \quad(0.6)$

$-\mathrm{c} .457 \mathrm{C}>\mathrm{T}$

c. $481 \mathrm{C}>\mathrm{T} \quad(0.6)$

c. $482 \mathrm{G}>\mathrm{A} \square(7.0)$

\begin{tabular}{l|l|l} 
c. 567 dup & & $(3.8)$ \\
\cline { 3 - 3 } &
\end{tabular}

c. $615 \mathrm{C}>\mathrm{A}(0.6)$

c. $616 \mathrm{C}>\mathrm{T}(0.6)$

658 660delAAG

NF

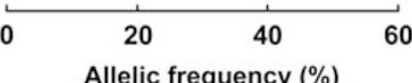

Figure 1 The $M M A C H C$ mutations found in 79 Chinese $c b / C$ MMA patients. (a) Genomic structure and (b) protein structure of the $M M A C H C$ gene predicted by Lerner-Ellis et al. ${ }^{2}$ Open and gray boxes are coding regions and untranslated regions, respectively. The black boxes represent the B12-binding domain and the TonB homolog. (c) Mutations identified in Chinese cb/C patients. Novel mutations are in bold. The Allelic frequency of each mutation is in parentheses. NF indicates that mutations were not found in the coding region, exon-intron boundary and untranslated region sequences.

strongly suggest that c.365A $>$ T (p.H122L) and c.452A $>$ G (p.H151R) might be disease-causing mutations.

Among the five novel frameshift mutations identified, the c.99delA, c.248dupT and c.626dupT mutations are predicted to cause a transla- tional frameshift and the creation of a premature stop codon. The insertion of an adenine nucleotide at cDNA position 445_446 (c.445_446insA) results in the substitution of a stop signal for a cysteine residue at codon 149 in the putative $\mathrm{B}_{12}$-binding domain. ${ }^{7}$ 
The c.277-3_303del30 mutation harbors a deletion across the boundary between intron 2 and exon 3, which ought to affect the splicing of the MMACHC transcript.

Of the 24 different mutations identified, 9 were identified in more than one family (Supplementary Table S1). Five of them, namely c.609G > A, c.658_660delAAG, c.482G > A, c.394C > T and c.80A $>$ G, were the most frequent found in these Chinese patients and accounted for $48.1 \%$ (76 of 158 ), $13.9 \%$ (22 of 158 ), $7 \%$ (11 of 158 ), $5.7 \%$ (9 of 158 ) and $5.1 \%$ (8 of 158) of disease alleles, respectively. The c.609G > A, c.658_660delAAG and c.394C $>$ T mutations have also been reported in other Chinese $c b l \mathrm{C}$ patients, ${ }^{10,12}$ which confirms the c.609G $>$ A and c.658_660delAAG mutations as the two most common mutations in Chinese patients. In addition, the c.609G $>$ A mutation accounted for $46.7 \%$ (70 of 150) and 75\% (6 of 8 ) of diseased alleles in Northern and Southern Chinese patients,

Table 2 SNPs identified in the MMACHC gene in a Chinese population

\begin{tabular}{|c|c|c|c|c|}
\hline \multirow{2}{*}{$\frac{\text { Variations }}{\text { c. } 1-302 \mathrm{~T}>\mathrm{G}}$} & \multirow{2}{*}{$\frac{\text { Location }}{5^{\prime} \text {-UTR }}$} & \multirow{2}{*}{$\begin{array}{c}\text { dbSNP no. } \\
\text { rs3748643 }\end{array}$} & \multicolumn{2}{|c|}{ Frequency } \\
\hline & & & $\mathrm{T}$ & $91.4 \%$ \\
\hline & & & $\mathrm{G}$ & $8.6 \%$ \\
\hline \multirow[t]{2}{*}{ c. $1-234 A>G$} & $5^{\prime}$-UTR & rs3748644 & A & $92.9 \%$ \\
\hline & & & $\mathrm{G}$ & $7.1 \%$ \\
\hline \multirow[t]{2}{*}{ c. $277-49 A>G$} & Intron 2 & & A & $94.3 \%$ \\
\hline & & & $\mathrm{G}$ & $5.7 \%$ \\
\hline \multirow[t]{2}{*}{ c. $321 \mathrm{G}>\mathrm{A}$} & Exon 3 & rs2275276 & $\mathrm{G}$ & $42.9 \%$ \\
\hline & & & A & $57.1 \%$ \\
\hline
\end{tabular}

Abbreviations: SNP, single-nucleotide polymorphism; UTR, untranslated region. respectively, indicating the major mutation profile of the $M M A C H C$ gene in Southern Chinese patients was not very different from that of Northern Chinese patients. The results suggest that the major mutation profile of the MMACHC gene was similar in Southern and Northern Chinese patients, although the incidence of the $c b l C$-type MMA in Southern Chinese might be lower than that in Northern Chinese.

In addition to the 24 mutations, 4 polymorphisms were identified in the sequenced region (exon 1-exon $4 \mathrm{a}$, Table 2). Three of them were known SNPs, namely c.1-302T $>$ G (rs3748643), c.1-234A > G (rs3748644) and c.321G >A (rs2275276, p.V107V) ${ }^{7}$ and one was a novel SNP, c.277-49A $>$ G, which was identified in intron 2. The allelic frequencies of c.1-302T $>$ G, c.1-234A $>$ G and c.321G $>$ A were similar to the frequencies among Chinese population reported in the HapMap project (http://snp.cshl.org/). ${ }^{27}$ The allelic frequencies of A and G for the c.277-49A > G SNP in the 70 anonymous Chinese controls were 94.7 and $5.3 \%$, respectively. These polymorphisms served as intragenic markers to construct haplotypes for further analysis to study whether these common MMACHC mutations were influenced by a founder effect in these Chinese patients. Table 3 lists the genotypes and estimated frequencies of each SNP haplotype. The haplotypes were designated from A to E. Among the Chinese controls, haplotype A (57\%) was the most common and haplotype $\mathrm{E}$ was the least frequent (1\%). The allelic distribution of a short tandem repeat marker upstream to the MMACHC gene, namely D1S2677, was also analyzed to aid in the study of the founder effect among common mutations. A total of nine alleles of D1S2677 were found in the normal population (Table 3). Among the normal population, the 137-bp allele was the most common genotype. No statistical significant differences were noted for the SNP haplotype frequencies and D1S2677 allelic distribution between the Southern and Northern Chinese controls (data not shown).

Table 3 Microsatellite and SNP haplotype frequencies in MMACHC mutant alleles and normal alleles

(a) SNP haplotypes in normal and mutant alleles

\begin{tabular}{|c|c|c|c|c|c|c|c|c|c|c|}
\hline \multirow[b]{2}{*}{ Haplotype } & \multicolumn{4}{|c|}{ Single-nucleotide polymorphism } & \multirow{2}{*}{$\begin{array}{l}\text { Control } \\
\mathrm{N}=140\end{array}$} & \multirow{2}{*}{$\begin{array}{c}c .80 A>G \\
N=7^{a}\end{array}$} & \multirow{2}{*}{$\begin{array}{c}\text { c. } 394 C>T \\
\mathrm{~N}=5^{\mathrm{a}}\end{array}$} & \multirow{2}{*}{$\begin{array}{c}c .482 G>A \\
N=8^{a}\end{array}$} & \multirow{2}{*}{$\begin{array}{c}c .609 G>A \\
N=65^{\mathrm{a}}\end{array}$} & \multirow{2}{*}{$\begin{array}{c}\text { c.658_660delAAG } \\
\mathrm{N}=11^{\mathrm{a}}\end{array}$} \\
\hline & c.1-302 & c. 1-234 & c. $277-49$ & c. 321 & & & & & & \\
\hline A & $\mathrm{T}$ & A & A & A & $80(57 \%)$ & $0(0 \%)$ & $3(60 \%)^{b}$ & $4(50 \%)^{b}$ & $0(0 \%)$ & $1(9 \%)$ \\
\hline$B$ & $\mathrm{~T}$ & A & $A$ & G & $40(29 \%)$ & $0(0 \%)$ & $1(20 \%)$ & $4(50 \%)^{b}$ & $0(0 \%)$ & $10(91 \%)^{c}$ \\
\hline C & G & G & A & $\mathrm{G}$ & $10(7 \%)$ & $7(100 \%)^{c}$ & $0(0 \%)$ & $0(0 \%)$ & $65(100 \%)^{c}$ & $0(0 \%)$ \\
\hline$D$ & $\mathrm{~T}$ & A & G & $\mathrm{G}$ & $8(6 \%)$ & $0(0 \%)$ & $1(20 \%)$ & $0(0 \%)$ & $0(0 \%)$ & $0(0 \%)$ \\
\hline$E$ & $\mathrm{G}$ & $A$ & $A$ & G & $2(1 \%)$ & $0(0 \%)$ & $0(0 \%)$ & $0(0 \%)$ & $0(0 \%)$ & $0(0 \%)$ \\
\hline
\end{tabular}

(b) Allelic distribution of D1S2677 marker in normal and mutant alleles

\begin{tabular}{|c|c|c|c|c|c|c|}
\hline D1S2677 & Controls & c. $80 A>G$ & c. $394 C>T$ & c. $482 G>A$ & c. $609 G>A$ & c.658_660delAAG \\
\hline Alleles (bp) & $N=514$ & $\mathrm{~N}=8^{\mathrm{a}}$ & $\mathrm{N}=9^{a}$ & $\mathrm{~N}=10^{\mathrm{a}}$ & $\mathrm{N}=70^{\mathrm{a}}$ & $\mathrm{N}=17^{\mathrm{a}}$ \\
\hline 133 & $0(0.0 \%)$ & $0(0.0 \%)$ & $0(0.0 \%)$ & $0(0.0 \%)$ & $12(17.1 \%)$ & $0(0.0 \%)$ \\
\hline 137 & 236 (45.9\%) & $6(75 \%)^{b}$ & $6(66.7 \%)^{b}$ & $9(90.0 \%)^{c}$ & $2(2.9 \%)$ & $13(76.4 \%)^{d}$ \\
\hline 139 & $10(1.9 \%)$ & $1(12.5 \%)$ & $0(0.0 \%)$ & $0(0.0 \%)$ & $56(80.0 \%)^{c}$ & 2 (11.8\%) \\
\hline 143 & 150 (29.2\%) & $1(12.5 \%)$ & 2 (22.2\%) & $0(0.0 \%)$ & $0(0.0 \%)$ & $1(5.9 \%)$ \\
\hline 145 & $46(8.9 \%)$ & $0(0.0 \%)$ & $1(11.1 \%)$ & 1 (10.0\%) & $0(0.0 \%)$ & $1(5.9 \%)$ \\
\hline Others ${ }^{\mathrm{e}}$ & $72(14.1 \%)$ & $0(0.0 \%)$ & $0(0.0 \%)$ & $0(0.0 \%)$ & $0(0.0 \%)$ & $0(0.0 \%)$ \\
\hline
\end{tabular}

Abbreviation: SNP, single-nucleotide polymorphism.

aAlleles of known phase. The phase was determined by a haplotype analysis of the unaffected parents and siblings.

bNot significant $(P>0.2)$ vs the normal Chinese population.

'Significantly different $(P<0.01)$ vs the normal Chinese population.

dNot significant, $(P=0.0234)$ vs the normal Chinese population.

eOther alleles included SNP lengths of $135 \mathrm{bp}(0.8 \%), 141 \mathrm{bp}(3.1 \%), 147 \mathrm{bp}(3.9 \%), 149 \mathrm{bp}(3.5 \%)$ and $151 \mathrm{bp}(2.7 \%)$ 
Among the common mutations, both c.80A $>\mathrm{G}$ (corrected $\chi^{2}=47.5, P<0.0001$, Table 3$)$ and c.609G $>$ A $\left(\chi^{2}=165.0, P<0.0001\right.$, Table 3) mutations were linked to the SNP haplotype $C$ and the c.658_660delAAG mutation was linked to the SNP haplotype B (corrected $\chi^{2}=15.2, P<0.0001$, Table 3 ). These results suggest that the spread of the c.80A $>$ G, c.609G $>$ A and c.658_660delAAG mutations may have undergone a founder effect in Chinese patients. In addition, the results of short tandem repeat analysis showed that each common mutation was linked to two to four different alleles of D1S2677. Most of the c.609G $>$ A alleles were linked to the 139-bp allele of D1S2677, whereas the majority of the c.80A $>\mathrm{G}$ and c.658_660delAAG alleles were linked to the 137-bp allele, which is the most common genotype found in the normal population (Table 3). Results from the $\chi^{2}$-test showed strong linkage disequilibrium between the c.609G $>$ A mutation and the 139-bp allele of D1S2677 $(P<0.0001)$, which further support a founder effect for the c.609G $>$ A mutation in Chinese patients. On the other hand, results from the $\chi^{2}$-test showed the absence of linkage between the 137-bp allele and the mutation c.80A $>$ G or c.658_660delAAG. A possible explanation for the observations of the different genotypes linked to the mutations is that founder mutations might have undergone subsequent multiple recombination events and/or slippage of microsatellite during transmission. This, in turn, suggests that the founder effect is not a recent event.

The results of the SNP haplotype and short tandem repeat analysis showed that the c.482G $>$ A and c.394C $>$ T mutations were not linked to any specific SNP haplotype (Table 3), but the majority of the two mutation alleles were linked to the 137-bp allele. The results also imply that the c.394C $>\mathrm{T}$ and $c .482 \mathrm{G}>\mathrm{A}$ mutations identified in these Chinese patients may come from several different origins. As the c.394C $>$ T and c.482G > A mutations occur at a CpG site and have also been observed in other populations, ${ }^{5,7,11-12,14}$ the recurrence of the c. $394 \mathrm{C}>\mathrm{T}$ and c. $482 \mathrm{G}>\mathrm{A}$ mutations in Chinese $c b l \mathrm{C}$ patients is probably due to a $\mathrm{CpG}$ mutation hotspot ${ }^{28}$ within the MMACHC gene rather than inherence of an ancestral allele. The CpG dinucleotide mutational hotspots within the $M M A C H C$ gene may also explain the recurrence of the c. $217 \mathrm{C}>\mathrm{T}, \mathrm{c} .457 \mathrm{C}>\mathrm{T}, \mathrm{c} .481 \mathrm{C}>\mathrm{T}$ and $\mathrm{c} .616 \mathrm{C}>\mathrm{T}$ mutations in Chinese patients and other populations. ${ }^{7,11-12}$

Clinically, two distinct phenotypes of the $c b l C$-type MMA have been classified according to the age of onset. ${ }^{2}$ In this study, information on age of onset was available for 71 patients (Table 4). ${ }^{16,18}$ Among the 71 patients, 55 early-onset patients presented with the disease in the first year of life, eight late-onset patients showed symptoms after 4 years of age and the other eight patients presented with symptoms at an age between 1 and 4 years. ${ }^{16,18}$

Information about age onset for 15 patients homozygous for the c.609G > A mutation was available (Table 4), including 12 early-onset patients and 3 patients presenting before the age of 4 years. The results suggest that the c.609G $>$ A mutation may be associated with the early-onset phenotype.

The eight late-onset patients presented with acute neurological deteriorations. However, after treatment, five of them recovered and showed normal levels of intelligence, whereas the remaining three improved significantly, but with some residual handicap. ${ }^{16}$ Among these eight late-onset $c b l C$ patients, seven patients $(87.5 \%)$ were compound heterozygous for the c. $482 \mathrm{G}>\mathrm{A}$ mutation and the remaining one was compound heterozygous for the c.452A>G and c.609G > A mutations. Although one patient, who was homozygous for the c.482G $>$ A mutation, presented with early-onset clinical symptoms (Table 4), the c.482G $>$ A mutation was in general associated with the late-onset phenotype and a milder clinical presenta-
Table 4 Age of onset of patients with various combinations of MMACHC mutations

\begin{tabular}{|c|c|c|c|c|c|}
\hline \multicolumn{2}{|c|}{ Genotype } & \multirow[b]{2}{*}{$\begin{array}{c}\text { No. of } \\
\text { patients }\end{array}$} & \multicolumn{3}{|c|}{ Age of onset (years) } \\
\hline Mutation & Mutation & & $<1$ & $1-4$ & $>4$ \\
\hline c. $609 \mathrm{G}>\mathrm{A}$ & c. $1 A>G$ & 3 & 3 & & \\
\hline c. $609 \mathrm{G}>\mathrm{A}$ & c. $80 A>G$ & 6 & 6 & & \\
\hline c. $609 \mathrm{G}>\mathrm{A}$ & c.146_154del9 & 1 & 1 & & \\
\hline c. $609 \mathrm{G}>\mathrm{A}$ & c. $217 \mathrm{C}>\mathrm{T}$ & 1 & 1 & & \\
\hline c. $609 \mathrm{G}>\mathrm{A}$ & c.248dupT & 1 & 1 & & \\
\hline c. $609 \mathrm{G}>\mathrm{A}$ & c.277-3_303del30 & 1 & 1 & & \\
\hline c. $609 \mathrm{G}>\mathrm{A}$ & c. $315 C>G$ & 1 & 1 & & \\
\hline c. $609 \mathrm{G}>\mathrm{A}$ & c.445_446insA & 1 & 1 & & \\
\hline c. $609 \mathrm{G}>\mathrm{A}$ & c. $452 A>G$ & 1 & & & 1 \\
\hline c. $609 \mathrm{G}>\mathrm{A}$ & c. $481 \mathrm{C}>\mathrm{T}$ & 1 & 1 & & \\
\hline c. $609 \mathrm{G}>\mathrm{A}$ & c.567dupt & 3 & 2 & 1 & \\
\hline c. $609 \mathrm{G}>\mathrm{A}$ & c. $609 \mathrm{G}>\mathrm{A}$ & 15 & 12 & 3 & \\
\hline c. $609 \mathrm{G}>\mathrm{A}$ & c. $616 \mathrm{C}>\mathrm{T}$ & 1 & 1 & & \\
\hline c. $609 \mathrm{G}>\mathrm{A}$ & c.626dupT & 1 & 1 & & \\
\hline c. $609 \mathrm{G}>\mathrm{A}$ & c.658_660delAAG & 8 & 8 & & \\
\hline c. $609 \mathrm{G}>\mathrm{A}$ & NF & 1 & 1 & & \\
\hline c.658_660delAAG & c. $80 A>G$ & 1 & 1 & & \\
\hline c.658_660delAAG & c. $365 \mathrm{~A}>\mathrm{T}$ & 1 & & 1 & \\
\hline c.658_660delAAG & c.567dupT & 2 & 2 & & \\
\hline c.658_660delAAG & $N F$ & 1 & 1 & & \\
\hline c. $482 \mathrm{G}>\mathrm{A}$ & c. $315 \mathrm{C}>\mathrm{G}$ & 1 & & & 1 \\
\hline c. $482 \mathrm{G}>\mathrm{A}$ & c. $482 \mathrm{G}>\mathrm{A}$ & 1 & 1 & & \\
\hline c. $482 \mathrm{G}>\mathrm{A}$ & c.567dupT & 1 & & & 1 \\
\hline c. $482 \mathrm{G}>\mathrm{A}$ & c. $609 \mathrm{G}>\mathrm{A}$ & 2 & & & 2 \\
\hline c. $482 \mathrm{G}>\mathrm{A}$ & c. $615 \mathrm{C}>\mathrm{A}$ & 1 & & & 1 \\
\hline c. $482 \mathrm{G}>\mathrm{A}$ & c.658_660delAAG & 3 & & 1 & 2 \\
\hline c. $394 \mathrm{C}>\mathrm{T}$ & c.99delA & 1 & 1 & & \\
\hline c. $394 \mathrm{C}>\mathrm{T}$ & c.398_399delAA & 1 & 1 & & \\
\hline c. $394 \mathrm{C}>\mathrm{T}$ & c. $609 \mathrm{G}>\mathrm{A}$ & 4 & 2 & 2 & \\
\hline c. $394 \mathrm{C}>\mathrm{T}$ & c.658_660delAAG & 2 & 2 & & \\
\hline c. $1 \mathrm{~A}>\mathrm{G}$ & c. $457 \mathrm{C}>\mathrm{T}$ & 1 & 1 & & \\
\hline c. $80 A>G$ & NF & 1 & 1 & & \\
\hline c.271dupA & c. $365 \mathrm{~A}>\mathrm{T}$ & 1 & 1 & & \\
\hline Total allele no. & & 71 & 55 & 8 & 8 \\
\hline
\end{tabular}

Patients without confirmed age-onset information were excluded.

tion. This observation is in accordance with previous reports. $5,7,11-12,14$ In addition to the c. $482 \mathrm{~A}>\mathrm{G}$ mutation, the newly identified c. $452 \mathrm{~A}>\mathrm{G}$ mutation was also seen in one late-onset patient, and thus this mutation may be also associated with late-onset and a mild phenotype. However, more patients carrying the c.452A $>\mathrm{G}$ are required to confirm this genotype-phenotype correlation.

It has been suggested that the c.394C $>\mathrm{T}$ mutation is associated with a late age of onset and may protect against developing an earlyonset phenotype. ${ }^{11}$ In this study, nine patients were compound heterozygous for the c.394C $>\mathrm{T}$ mutation $(5.7 \%)$. None of the nine patients showed late onset (Table 4). In these circumstances, it is unclear whether the second mutations, namely c.99delA, c.389_399delAA, c.658_660delAAG and c.609G > A, may have a dominant negative effect and cause the early-onset phenotype, or that interactions between various other factors, such as genetic background, environmental influences and dietary habits, have had a 
role in the clinical presentation. ${ }^{29-33}$ In this context, further research is required to elucidate the genotype-phenotype correlation of the c.394C $>$ T mutation.

In conclusion, our study has identified the genotype of 79 Chinese patients with combined MMA and HC, as well as measuring allelic frequencies of the $M M A C H C$ mutations in these patients. The results indicate that defects occurring in the MMACHC gene are the major cause of combined MMA and HC in these Chinese patients. In Chinese $c b l \mathrm{C}$ patients, the c.609G $>\mathrm{A}$, c.658_660delAAG, c. $482 \mathrm{G}>\mathrm{A}, \mathrm{c} .394 \mathrm{C}>\mathrm{T}$ and c. $80 \mathrm{~A}>\mathrm{G}$ mutations accounted for $80 \%$ of disease alleles. On the basis of the fact that the most common types of MMACHC mutations in Chinese have been elucidated, direct mutation analysis can therefore be used as a rapid confirmatory diagnostic method for Chinese patients with combined MMA and HC. This approach can also be applied for carrier detection as part of genetic counseling and as a prenatal diagnosis tool.

\section{ACKNOWLEDGEMENTS}

We thank the Sequencing Core of Genome Research Center of the National Yang-Ming University for sequencing works and Dr Tsai, S.-F. for discussions and various helpful suggestions. This work was partially supported by the National Health Research Institutes and grants from the Bureau of Health Promotion, Department of Health (DOH94-HP-2204 and DOH95-HP-2206), Taiwan, Republic of China.

1 Fowler, B., Leonard, J. V. \& Baumgartner, M. R. Causes of and diagnostic approach to methylmalonic acidurias. J. Inherit. Metab. Dis. 31, 350-360 (2008).

2 Rosenblatt, D. S., Aspler, A. L., Shevell, M. I., Pletcher, B. A., Fenton, W. A. \& Seashore, M. R. Clinical heterogeneity and prognosis in combined methylmalonic aciduria and homocystinuria (cblC). J. Inherit. Metab. Dis. 20, 528-538 (1997).

3 Bodamer, O. A., Rosenblatt, D. S., Appel, S. H. \& Beaudet, A. L. Adult-onset combined methylmalonic aciduria and homocystinuria (cblC). Neurology 56, 1113 (2001).

4 Roze, E., Gervais, D., Demeret, S., Ogier de Baulny, H., Zittoun, J., Benoist, J. F. et al. Neuropsychiatric disturbances in presumed late-onset cobalamin C disease. Arch. Neurol. 60, 1457-1462 (2003)

5 Tsai, A. C., Morel, C. F., Scharer, G., Yang, M., Lerner-Ellis, J. P., Rosenblatt, D. S. et al. Late-onset combined homocystinuria and methylmalonic aciduria (cblC) and neuropsychiatric disturbance. Am. J. Med. Genet. A 143A, 2430-2434 (2007).

6 Thauvin-Robinet, C., Roze, E., Couvreur, G., Horellou, M. H., Sedel, F., Grabli, D. et al. The adolescent and adult form of cobalamin $C$ disease: clinical and molecular spectrum. J. Neurol. Neurosurg. Psychiatry 79, 725-728 (2008).

7 Lerner-Ellis, J. P., Tirone, J. C., Pawelek, P. D., Dore, C., Atkinson, J. L., Watkins, D. et al. Identification of the gene responsible for methylmalonic aciduria and homocystinuria, cbIC type. Nat. Genet. 38, 93-100 (2006).

$8 \mathrm{Kim}$, J., Gherasim, C. \& Banerjee, R. Decyanation of vitamin B12 by a trafficking chaperone. Proc. Natl Acad. Sci. USA 105, 14551-14554 (2008).

9 Heil, S. G., Hogeveen, M., Kluijtmans, L. A., van Dijken, P. J., van de Berg, G. B., Blom, H. J. et al. Marfanoid features in a child with combined methylmalonic aciduria and homocystinuria (CbIC type). J. Inherit. Metab. Dis. 30, 811 (2007).

10 Yuen, Y. P., Lai, C. K., Chan, Y. W., Lam, C. W., Tong, S. F. \& Chan, K. Y. DNA-based diagnosis of methylmalonic aciduria and homocystinuria, cblC type in a Chinese patient presenting with mild developmental delay. Clin. Chim. Acta. 375, 171-172 (2007).
11 Nogueira, C., Aiello, C., Cerone, R., Martins, E., Caruso, U., Moroni, I. et al. Spectrum of MMACHC mutations in Italian and Portuguese patients with combined methylmaIonic aciduria and homocystinuria, cblC type. Mol. Genet. Metab. 93, 475-480 (2008).

12 Lerner-Ellis, J. P., Anastasio, N., Liu, J., Coelho, D., Suormala, T., Stucki, M. et al. Spectrum of mutations in MMACHC, allelic expression, and evidence for genotypephenotype correlations. Hum. Mutat. 30, 1072-1081 (2009).

13 Tang, H., Hao, H., Tang, S. H., Chen, X., Liu, F., Cha, Q. B. et al. Mutation analysis of the MMACHC gene in a pedigree with methylmalonic aciduria. Chin. J. Med. Genet. 26, 62-65 (2009). (in Chinese).

14 Morel, C. F., Lerner-Ellis, J. P. \& Rosenblatt, D. S. Combined methylmalonic aciduria and homocystinuria (cblC): phenotype-genotype correlations and ethnic-specific observations. Mol. Genet. Metab. 88, 315-321 (2006).

15 Hori, D., Hasegawa, Y., Kimura, M., Yang, Y., Verma, I. C. \& Yamaguchi, S. Clinical onset and prognosis of Asian children with organic acidemias, as detected by analysis of urinary organic acids using GC/MS, instead of mass screening. Brain. Dev. 27, 39-45 (2005).

16 Yang, Y., Sun, F., Song, J., Hasegawa, Y., Yamaguchi, S., Zhang, Y. et al. Clinical and biochemical studies on Chinese patients with methylmalonic aciduria. J. Child. Neurol. 21, 1020-1024 (2006).

17 Niu, D. M., Chien, Y. H., Chian, C. C., Ho, H. C., Hwu, W. L., Kao, S. M. et al. National survey of extended newborn screening by tandem mass spectrometry in Taiwan. J. Inherit. Metab. Dis. (2010), doi:10.1007/s10545-010-9129-z

18 Zhang, Y., Song, J. Q., Liu, P., Yan, R., Dong, J. H., Yang, Y. L. et al. Clinical studies on fifty-seven Chinese patients with combined methylmalonic aciduria and homocysteinemia. Chin. J. Pediatr. 45, 513-517 (2007). (in Chinese).

19 Zhang, Y., Yang, Y. L., Hasegawa, Y., Yamaguchi, S., Shi, C. Y., Song, J. Q. et al. Prenatal diagnosis of methylmalonic aciduria by analysis of organic acids and total homocysteine in amniotic fluid. Chin. Med. J. 121, 216-219 (2008).

20 Liu, T. T., Hsiao, K. J., Lu, S. F., Wu, S. J., Wu, K. F., Chiang, S. H. et al. Mutation analysis of the 6-pyruvoyl-tetrahydropterin synthase gene in Chinese hyperphenylalaninemia caused by tetrahydrobiopterin synthesis deficiency. Hum. Mutat. 11, 76-83 (1998).

21 Hong, C. J., Song, H. L., Lai, H. C., Tsai, S. J. \& Hsiao, K. J. Methanol/acetone treatment helps the amplification of FMR1 CGG repeat fragment in dried blood spots from Guthrie cards. Lancet 353, 1153-1154 (1999).

22 Gordon, D., Abajian, C. \& Green, P. Consed: a graphical tool for sequence finishing. Genome Res. 8, 195-202 (1998).

23 Nickerson, D. A., Tobe, V. O. \& Taylor, S. L. PolyPhred: automating the detection and genotyping of single nucleotide substitutions using fluorescence-based resequencing. Nucleic Acids Res. 25, 2745-2751 (1997).

24 den Dunnen, J. T. \& Antonarakis, S. E. Mutation nomenclature extensions and suggestions to describe complex mutations: a discussion. Hum. Mutat. 15, 7-12 (2000).

25 Zhao, J. H. 2LD, GENECOUNTING and HAP: computer programs for linkage disequilibrium analysis. Bioinformatics 20, 1325-1326 (2004).

26 Zhao, J. H., Lissarrague, S., Essioux, L. \& Sham, P. C. GENECOUNTING: haplotype analysis with missing genotypes. Bioinformatics 18, 1694-1695 (2002).

27 The International HapMap Consortium. The International HapMap project. Nature 426, 789-796 (2003).

28 Cooper, D. N. \& Youssoufian, H. The CpG dinucleotide and human genetic disease. Hum. Genet. 78, 151-155 (1988).

29 Dipple, K. M. \& McCabe, E. R. Modifier genes convert 'simple' Mendelian disorders to complex traits. Mol. Genet. Metab. 71, 43-50 (2000).

30 Dipple, K. M. \& McCabe, E. R. Phenotypes of patients with 'simple' Mendelian disorders are complex traits: thresholds, modifiers, and systems dynamics. Am. J. Hum. Genet. 66, 1729-1735 (2000).

31 Dipple, K. M., Phelan, J. K. \& McCabe, E. R. Consequences of complexity within biological networks: robustness and health, or vulnerability and disease. Mol. Genet. Metab. 74, 45-50 (2001).

32 Scriver, C. R. \& Waters, P. J. Monogenic traits are not simple: lessons from phenylketonuria. Trends Genet. 15, 267-272 (1999).

33 Vockley, J., Rinaldo, P., Bennett, M. J., Matern, D. \& Vladutiu, G. D. Synergistic heterozygosity: disease resulting from multiple partial defects in one or more metabolic pathways. Mol. Genet. Metab. 71, 10-18 (2000).

Supplementary Information accompanies the paper on Journal of Human Genetics website (http://www.nature.com/jhg) 